\title{
Comparison of activity indexes for recognizing enzyme mutants of higher activity with uricase as model
}

Juan Feng ${ }^{\dagger}$, Hongbo Liư ${ }^{\dagger}$ Xiaolan Yang ${ }^{\dagger}$, Ang Gao, Juan Liao, Liping Feng, Jun Pu, Yanling Xie, Gaobo Long, Yuanli Li and Fei Liao*

\begin{abstract}
Background: For screening a library of enzyme mutants, an efficient and cost-effective method for reliable assay of enzyme activity and a decision method for safe recognition of mutants of higher activity are needed. The comparison of activity concentrations of mutants in lysates of transformed Escherichia coli cells against a threshold is unsafe to recognize mutants of higher activity due to variations of both expression levels of mutant proteins and lysis efficiency of transformed cells. Hence, by a spectrophotometric method after verification to measure uricase activity, specific activity calculated from the level of total proteins in a lysate was tested for recognizing a mutant of higher activity.

Results: During uricase reaction, the intermediate 5-hydroxyisourate interferes with the assay of uric acid absorbance, but the measurement of absorbance at $293 \mathrm{~nm}$ in alkaline borate buffer was reliable for measuring uricase initial rates within a reasonable range. The level of total proteins in a lysate was determined by the Bradford assay. Polyacrylamide gel electrophoresis analysis supported different relative abundance of uricase mutant proteins in their lysates; activity concentrations of uricase in such lysates positively correlated with levels of total proteins. Receiver-operation-curve analysis of activity concentration or specific activity yielded area-under-the-curve close to 1.00 for recognizing a mutant with $>200 \%$ improvement of activity. For a mutant with just about $80 \%$ improvement of activity, receiver-operation-curve analysis of specific activity gave area-under-the-curve close to 1.00 while the analysis of activity concentration gave smaller area-under-the-curve. With the mean plus 1.4-fold of the standard deviation of specific activity of a starting material as the threshold, uricase mutants whose activities were improved by more than $80 \%$ were recognized with higher sensitivity and specificity.
\end{abstract}

Conclusion: Specific activity calculated from the level of total proteins is a favorable index for recognizing an enzyme mutant with small improvement of activity.

Keywords: Activity concentration, Interference, Positive candidate, Specific activity, Spectrometric methods, Threshold, Uricase

\footnotetext{
* Correspondence: liaofeish@yahoo.com

${ }^{\dagger}$ Equal contributors

Unit for Analytical Probes and Protein Biotechnology, Key Laboratory of

Clinical Laboratory Diagnostics of the Education Ministry, College of

Laboratory Medicine, Chongqing Medical University, Chongqing 400016,

China
} 


\section{Background}

Evolution biotechnology is powerful to discover new enzymes in chemical biology [1-4]. The practice of evolution biotechnology requires an efficient and cost-effective analytical method for reliable assay of catalytic capacities of mutants, and a suitable decision method for recognizing mutants of higher catalytic capacity as positive candidates or potential hits. In general, there should be mutagenesis on multiple sites of an enzyme to significantly improve the catalytic capacity; random mutagenesis of all possible sites of an enzyme will produce a huge library to burden the screening process. Hence, the design of focused libraries of mutants of an enzyme via mutagenesis of just a few sites is favorable, but a suitable decision method is required to recognize positive candidates with small improvement of catalytic capacity [5-8].

Catalytic capacity of an enzyme is measurable only with its sample of the highest purity. To practice the screening of a library, however, crude enzymes of mutants in lysates of transformed Escherichia coli cells after induced expression have to be employed as samples to measure activities for comparison against a suitable threshold derived from that of the starting material [6-13]. In general, the concentration of an enzyme in a lysate reflected by its activity (denoted as activity concentration hereafter) or specific activity can be utilized as activity index for comparison. The comparison of activity concentration of a mutant in its lysate against a threshold to recognize positive candidates is utilized in classical decision methods, but the variations in levels of mutant proteins due to differences in the inoculation quantity of a clone, amplification efficiency of the clone, induced expression levels and/or lysis efficiency of transformed cells reduce the safety for recognizing positive candidates. The comparison of specific activity may facilitate recognizing a mutant of small improvement of activity, but is prevented from practicing by the lack of efficient methods for selective assay of a mutant protein in its lysate. Alternatively, the quantification of total proteins in a lysate by a conventional protein assay may yield a practical specific activity to facilitate recognizing a positive candidate, but the effectiveness of this approach has not been reported yet.

Uricases are biodrugs to treat refractory gout [14-17], and pivotal analytical tools to measure serum uric acid [18]. Any application of uricase requires its catalytic capacity as high as possible [19-21], but available wildtype uricases all have low catalytic capacity. To date, uricase catalytic mechanisms are unknown and evolution biotechnology is practical to obtain uricase mutants of higher catalytic capacity. Uricase catalyzes the oxidation of uric acid into hydrogen peroxide and 5-hydroxyisourate (HIU) that spontaneously decomposes into allantoin of no absorbance at wavelengths over $280 \mathrm{~nm}$ [22]. Peroxidase- coupled and ferrous/ferric ion-mediated assays of hydrogen peroxide can measure uricase activity by quantifying absorbance or fluorescence [23-27], but require the termination of uricase reaction and thus reduce efficiency for screening uricase mutants. Notably, there must be a large portion of uric acid leftover in reaction solution to measure uricase initial rates; residual uric acid in uricase reaction solutions usually interferes with such methods for quantifying hydrogen peroxide $[28,29]$. On the other hand, uric acid has a strong absorbance peak at $293 \mathrm{~nm}$ and the assay of uric acid absorbance has the highest efficiency and the lowest cost for screening uricase mutants. However, HIU has an absorption peak around $302 \mathrm{~nm}$ and may interfere with the assay of uricase initial rates by measuring uric acid absorbance [30,31]. Therefore, the screening of uricase mutants needs a decision method to safely recognize positive candidates and the verification of the assay of uric acid absorbance for measuring uricase activity.

The uricase of Bacillus fastidious is a promising biodrug for treating refractory gout and a suitable tool for serum uric acid assay [32-34]; we cloned this uricase gene and obtained some mutants with small differences in catalytic capacities. In this report, the assay of uric acid absorbance at $293 \mathrm{~nm}$ was verified for measuring uricase activity; specific activity calculated from the level of total proteins in a lysate was tested as the index for comparison against a threshold for recognizing a positive candidate.

\section{Materials and methods}

\section{Reagents, chemicals and apparatus}

Uric acid was from Sigma-Aldrich. Boric acid, tri(hydroxylmethyl)-aminomethane (Tris), and sodium borate were common reagents of analytical grade or better. Water was re-stilled before use. $p$ ET28a vector carrying on the coding sequence for the wildtype intracellular uricase from Bacillus fastidious A.T.C.C. 29604 was that we used previously (gene id FJ393559, protein id ACR09749.1) [33]. By site-directed mutagenesis or the substitution of a fragment with an intended sequence, uricase mutants with different sequences of the $\mathrm{N}$-terminus and/or mutagensis of the unique cysteine residue into aspartic acid residue were generated (Additional file 1: sheet 1, mutant sequences and summary; these vectors were constructed via technical service provided by Taihe Biotechnolyg Co. Ltd, Beijing 100070, China). Escherichia coli cell strain BL21 (DE3) as the host to harvest vectors of such uricases for induced expression was provided by Sangon Biotechnology (Shanghai, China). DEAE-cellulose was from Whatman (Kent, UK). Xinmao UV 7504 spectrophotometer (http://www.china-xinmao.com) was used throughout the work. 


\section{Recombinant expression and purification of the wildtype uricase}

The expression of the wildtype uricase followed that described previously [33]. After the induced expression with isopropyl- $\beta-D$-thiogalactoside (IPTG) for $18 \mathrm{~h}$ at $16^{\circ} \mathrm{C}$, Escherichia coli cells BL21 (DE3) were harvested, and lysed by sonication; the soluble uricase was purified over two consecutive DEAE-cellulose chromatography via the elution with $0.10 \mathrm{M}$ Tris- $\mathrm{HCl}$ at $\mathrm{pH} 8.0$ plus a linear gradient of $\mathrm{NaCl}$ from 0 to $0.40 \mathrm{M}$. Fractions with the activities over $6.0 \mathrm{U} \cdot \mathrm{mg}^{-1}$ protein were pooled, and dialyzed against $50.0 \mathrm{mM}$ sodium borate buffer $\left(\mathrm{Na}_{2} \mathrm{~B}_{4} \mathrm{O}_{7} \cdot 10 \mathrm{H}_{2} \mathrm{O}\right)$ at $\mathrm{pH} 9.2$ for $24 \mathrm{~h}$ at $4^{\circ} \mathrm{C}$ with several changes of the buffer, and then stored at $4^{\circ} \mathrm{C}$ before use. Uricase activities were calibrated at $25^{\circ} \mathrm{C}$ with $0.075 \mathrm{mM}$ uric acid in borate buffer at $\mathrm{pH} 9.2$ by measuring absorbance at $293 \mathrm{~nm}$. One unit of uricase oxidized one micromole uric acid per min calculated with the extinction coefficient of $11.5(\mathrm{mM})^{-1} \cdot \mathrm{cm}^{-1}[33,35]$.

\section{Monitor of uricase reaction and estimation of Michaelis- Menten constant}

Borate buffer was prepared with a required ratio of 50.0 $\mathrm{mM}$ sodium borate $\left(\mathrm{Na}_{2} \mathrm{~B}_{4} \mathrm{O}_{7} \cdot 10 \mathrm{H}_{2} \mathrm{O}\right)$ to $0.20 \mathrm{M}$ boric acid [35]. Each reaction mixture in a total of $0.80 \mathrm{~mL}$ contained $50 \mu \mathrm{L}$ uricase solution and $0.75 \mathrm{ml}$ solution of uric acid in the borate buffer at an indicated $\mathrm{pH}$. The solutions of uric acid were pre-incubated at $(25 \pm 0.5)^{\circ} \mathrm{C}$ for 20 min before use and prepared every day. Reaction was initiated by the addition of uricase solution; absorbance was recorded in an isolated small room air-conditioned at $25^{\circ} \mathrm{C}$, after a lag time of $20 \mathrm{~s}$ at proper intervals within 5 min, unless otherwise stated [36,37]. The wildtype intracellular uricase of Bacillus fastidious A.T.C.C. 29604 has Michaelis-Menten constant $\left(K_{\mathrm{m}}\right)$ over $0.20 \mathrm{mM}$ and uric acid over $0.40 \mathrm{mM}$ is needed for estimating $K_{\mathrm{m}}$ [30,31]; the uricase of Asahi-Kasei (http://www.asahi-kasei.co.jp/ shindan/eng/list/index.html) has $K_{\mathrm{m}}$ below $50 \mu \mathrm{M}$ and the use of uric acid below $0.10 \mathrm{mM}$ is sufficient for estimating its $K_{\mathrm{m}}$. Hence, to test the estimation of $K_{\mathrm{m}}$, the absorbance of uric acid was measured at $308 \mathrm{~nm}$ with the recombinant wildtype uricase of Bacillus fastidious A.T.C.C. 29604, but at $293 \mathrm{~nm}$ with the uricase from Asahi-Kasei, for the maximal absorbance of uric acid within the measurable range of the spectrophotometer. Initial rates were average velocities when uric acid consumption percentages were below $10 \%$ after $40 \mathrm{~s}$ since reaction initiation. $K_{\mathrm{m}}$ was estimated by regression analyses according to LineweaverBurk plot with determination coefficients over 0.97.

\section{Assay of total proteins}

Protein quantity in a lysate was measured by the Bradford method [38]. All operations completely followed those described in the publication. Specific activity was calculated with the level of total proteins in a lysate as determined.

\section{Operation procedure for screening uricase mutants}

After purification via two consecutive DEAE-Cellulose chromoatography followed by preparative polyacrylamide gel electrophoresis (PAGE), uricase catalytic capacity was the highest specific activity determined with $75 \mu \mathrm{M}$ uric acid in the borate buffer at $\mathrm{pH}$ 9.2. Four uricase mutants of the bacterial uricase as active homotetramers were utilized and denoted as candidate A, B, C and D with their catalytic capacities in a descent order. Among those four candidates, any two uricases were randomly selected into a pair. For such pairs of uricases, the ratios of their catalytic capacities ranged from about 1.3 to about 4.0. Their sequences and kinetic parameters were provided in Additional file 1 (sheet 1 , mutant sequences and summary).

To mimic the procedure of screening, the pET28a vector of each mutant was transformed into Escherichia coli BL21 (DE3) cells. From each vector, a total of 30 clones on a selective plate with karnamycin were picked one-by-one; every clone was transferred into $1.0-\mathrm{mL}$ selective medium in a $5.0-\mathrm{ml}$ Ependorf tube for cultivation at $37^{\circ} \mathrm{C}$ for $3 \mathrm{~h}$. Then, IPTG for final $1.0 \mathrm{mM}$ was added to induce the expression of uricase for $18 \mathrm{~h}$ at $16^{\circ} \mathrm{C}$. Cells from each tube were harvested by centrifugation and lyzed by sonication treatment in ice-water bath at $150 \mathrm{~W}$ for $2.0 \mathrm{~min}$ (continuous treatment for $2.5 \mathrm{~s}$ at intervals of $2.5 \mathrm{~s}$, Ningbo Xinzhi Biomedicine LTD, Zhejiang, China; http://www.csb17.cn/Photo_Show.asp? InfoId=231, model JY92-II sonics cell lysis equipment); insoluble materials were removed by centrifugation at 10,000 rpm for $10 \mathrm{~min}$ again. The untransformed $E$. coli BL21 cells contained no endogenous uricase and thus activities of uricases in cell lysates were directly measured. In each clear lysate, uricase activity after proper dilution was measured in duplicate by monitoring UV absorbance at $293 \mathrm{~nm}$ with $75 \mu \mathrm{M}$ uric acid in $0.20 \mathrm{M}$ sodium borate buffer at $\mathrm{pH}$ 9.2. The dilution ratio for each candidate was optimized. Abundance of uricase protein in such lysates was examined by SDS-PAGE followed by staining proteins, and further by PAGE to visualize the active forms of uricases via the staining of activity (the staining reaction produces the insoluble visible chromogen through the coupled peroxidase action on a chromogenic substrate and hydrogen peroxide released by the action of uricase on uric acid) [39].

\section{Data processing and recognition of a mutant of higher activity}

Coefficient of variation (CV) was deduced from standard deviation (SD) and mean, and was compared by $F$-test to examine homoscedasticity. Statistical comparisons and 
correlation analyses were made with MS Excel 6.0. Normality of data was examined with Shapiro-Wilk test incorporated in SPSS 17.0.

In each pair of two uricases, the one of lower catalytic capacity is taken as the starting material and the other of higher catalytic capacity is designated as the positive candidate. Two methods were used for the recognition of a positive candidate in a pair with its activity measured in a lysate. Firstly, statistical comparison of averages of activity indexes in lysates of two uricases in a pair was used. Secondly, data in each pair were analyzed by receiver-operation-curve (ROC) analysis with SPSS 17.0 to determine the area-under-the-curve (AUC) [40,41]. The response of AUCs to ratios of catalytic capacities of paired uricases was examined. The minimal ratio in the catalytic capacities of a pair of uricases for an AUC of about 1.00 was selected; from ROC of this pair of two uricase, a threshold was selected for the sensitivity of $90 \%$ and specificity as close to $90 \%$ as possible. Such a threshold was normalized with respect to the mean and SD of the starting material to test its universal applicability to other pairs of uricases.

\section{Results and discussion}

Verification of the assay of uric acid absorbance for measuring initial rate

For screening mutants, uricase activities are reflected by change rates of uric acid absorbance during initial rate reaction. The interference of HIU with the assay of uricase initial rate by measuring uric acid absorbance depends on extinction coefficient and dynamic levels of
HIU. When uric acid consumption percentages are low enough for initial rate reaction, there will be no interference from HIU with the assay of uricase initial rate and thus a linear decrease of uric acid absorbance if HIU levels remain steady, or else, there will be interference from HIU with the assay of uricase initial rate and thus a nonlinear decrease of uric acid absorbance. Steadystate level of HIU and its lag time are determined by its production and decomposition rates. Steady-state of uricase reaction requires a lag time of about $40 \mathrm{~s}[35,36]$. When the consumption of uric acid is within $10 \%$, nonlinear decrease of uric acid absorbance after a lag time of $40 \mathrm{~s}$ directly supports that HIU levels do not achieve steady-state. If steady-state of HIU is achieved after so long lagging periods that the consumption percentages of uric acid exceed the limits for initial rate reaction, there will be inaccurate initial rates of uricases and such deviations are more pronounced with uricases of higher $K_{\mathrm{m}}$. Any factor affecting the production and decomposition rates of HIU including uricase activity, the use of Tris- $\mathrm{HCl}$ buffer or borate buffer, and reaction $\mathrm{pH}$, will alter the steady-state levels of HIU and thus its interference with the assay of uricase initial rate by measuring uric acid absorbance [30,31]. On the other hand, the wavelength to quantify uric acid absorbance also alters extinction coefficient of HIU and thus its interference with the assay of uricase initial rates. The absorbance peak of uric acid locates at $293 \mathrm{~nm}$ while that of HIU is around $302 \mathrm{~nm}[30,31]$. The bacterial uricase has $K_{\mathrm{m}}$ over $0.20 \mathrm{mM}$ for uric acid [32,33]; the highest level of uric acid should be about $0.4 \mathrm{mM}$ to
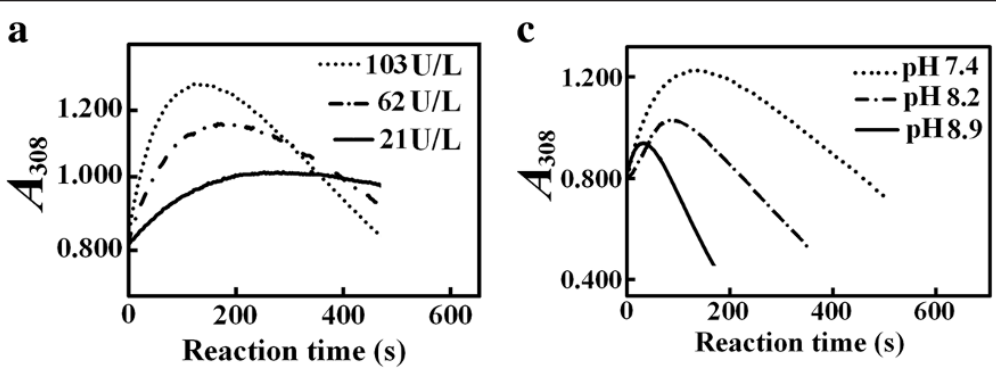

b

d
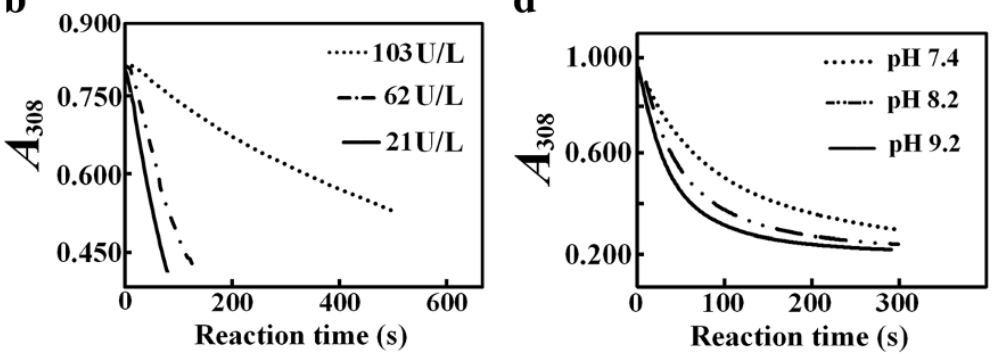

Figure 1 Interference from HIU with the assay of absorbance at $308 \mathrm{~nm}$ after the lag time of $10 \mathrm{~s}$ for $0.30 \mathrm{mM}$ uric acid under uricase action at $25^{\circ} \mathbf{C}$. a. Effects of activities on interference in $0.10 \mathrm{M}$ Tris- $\mathrm{HCl}$ buffer at pH $7.4 \mathbf{~ b}$. Effects of activities on interference in $0.20 \mathrm{M}$ borate buffer at pH 7.4 c. Effects of reaction pH on interference in $0.10 \mathrm{M}$ Tris- $\mathrm{HCl}$ buffer with final uricase activity at $103 \mathrm{U} / \mathrm{L} \mathbf{d}$. Effects of reaction pH on interference in $0.20 \mathrm{M}$ borate buffer with final uricase activity at $103 \mathrm{U} / \mathrm{L}$. 


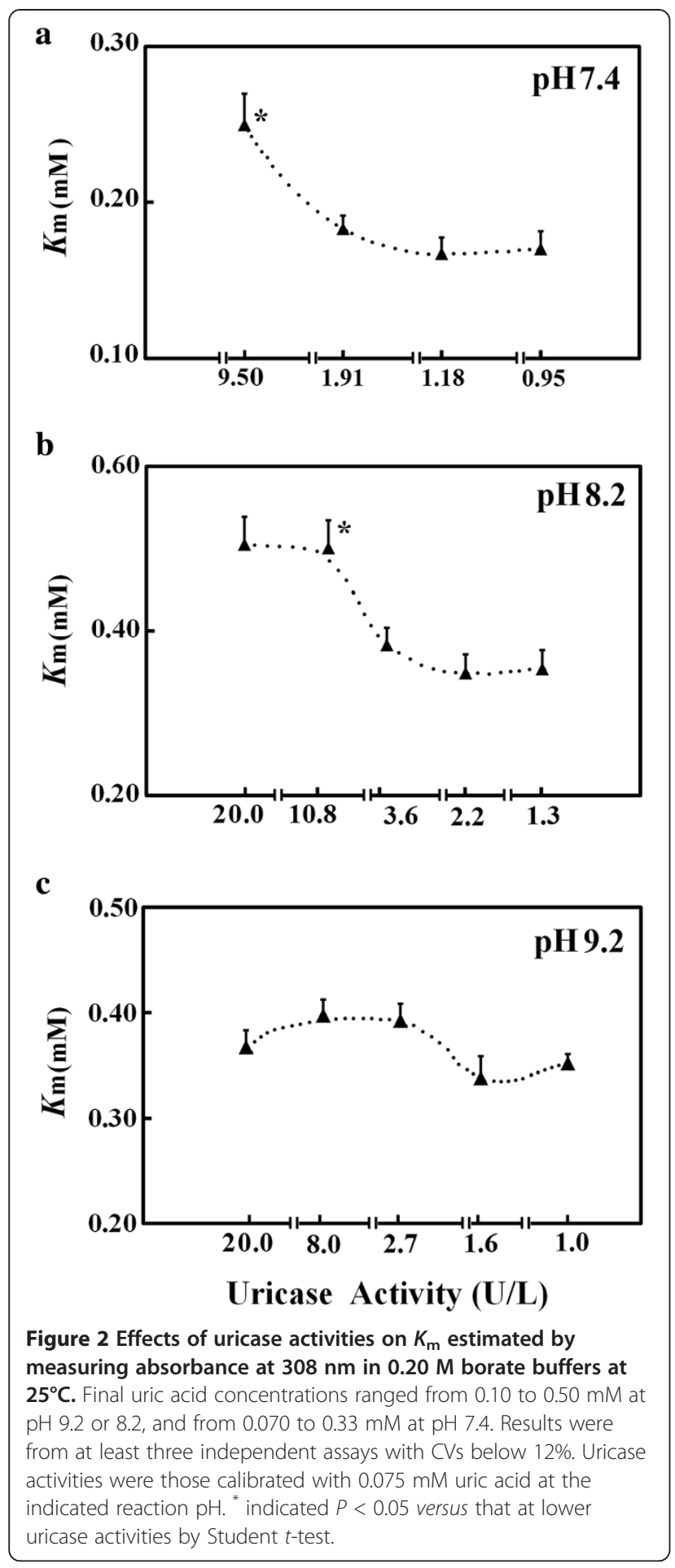

estimate its $K_{\mathrm{m}}$. Only at wavelengths no shorter than $308 \mathrm{~nm}$, absorbance for uric acid at $0.4 \mathrm{mM}$ is below 1.200 and measurable with common spectrophotometers; the interference from HIU with the assay of uricase initial rate by measuring uric acid absorbance should be more manifest at $308 \mathrm{~nm}$ than at $293 \mathrm{~nm}$. The bacterial uricase is thus an ideal model to test the existence and

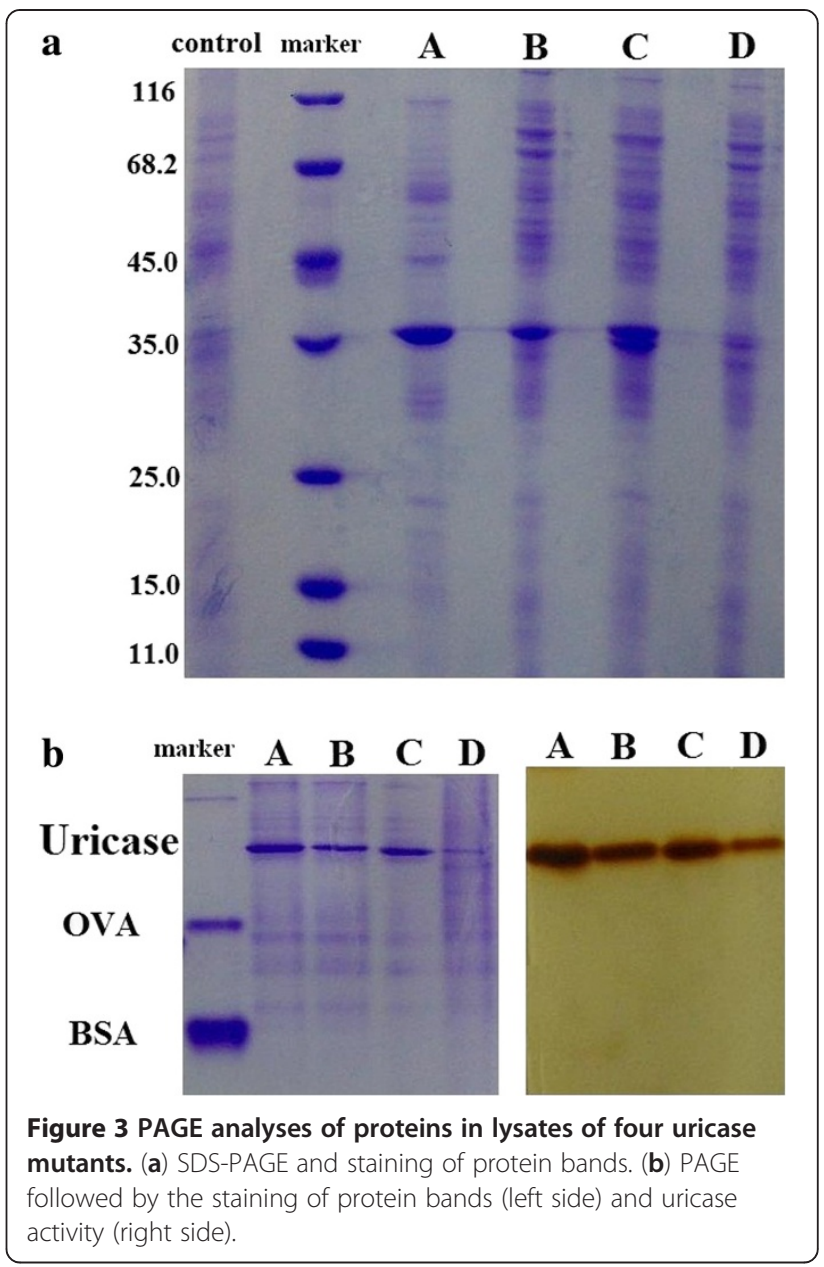

solution of the interference from HIU with the assay of uricase initial rate. Moreover, uricase activities will alter the interference from HIU with initial rates and thus $K_{\mathrm{m}}$ estimated via Lineweaver-Burk plot analysis of initial rates, which violates the facts with common enzymes. Hence, the interference from HIU with the assay of uricase initial rates by uric acid absorbance is examined based on: (a) effects of uricase activities, reaction buffers and $\mathrm{pH}$ values on nonlinear decreases of absorbance of uric acid when its consumption percentages are below $10 \%$, (b) effects of wavelengths to quantify uric acid absorbance on nonlinear decreases of absorbance of uric acid when its consumption percentages are below $10 \%$, and (c) the effects of uricase activities on $K_{\mathrm{m}}$.

In Tris- $\mathrm{HCl}$ buffer at 7.4, the interference from $\mathrm{HIU}$ with the absorbance of $0.30 \mathrm{mM}$ uric acid at $308 \mathrm{~nm}$ was detected as a continuous increase rather than decrease in absorbance till a peak at about $100 \mathrm{~s}$ since reaction initiation, even the consumption of uric acid was within 10\%; the use of a higher uricase activity produced a larger increase in absorbance at $308 \mathrm{~nm}$ after $100 \mathrm{~s}$ since reaction initiation (Figure 1). These results 
Table 1 Uricase activities at $0.075 \mathrm{mM}$ uric acid and protein levels in lysates $(n=30)$

\begin{tabular}{lcccc}
\hline Candidates/parameters & A & B & C & D \\
\hline Total proteins by the Bradford assay & 0.63 & 0.66 & 0.65 & 0.66 \\
& $(0.09)$ & $(0.10)$ & $(0.15)$ & $(0.09)$ \\
Activity concentration & 0.30 & 0.28 & 0.18 & 0.033 \\
& $(0.07)$ & $(0.06)$ & $(0.05)$ & $(0.004)$ \\
P for normality of AC by & 0.104 & 0.182 & 0.439 & 0.282 \\
shapiro-Wilk test & & & & \\
Correlation coefficient (R) & $>0.63$ & $>0.81$ & $>0.84$ & $>0.44$ \\
Specific activity & 0.49 & 0.43 & 0.28 & 0.050 \\
& $(0.09)$ & $(0.05)$ & $(0.05)$ & $(0.006)$ \\
$P$ for normality of SA by Shapiro-Wilk test & $<0.001$ & 0.310 & 0.972 & 0.031
\end{tabular}

Numbers in parentheses were SD. Correlation coefficient was that for the association of activity concentrations with levels of total proteins. $R>0.361$ and $>0.464$ indicated $P<0.05$ and $P<0.01$ for the association of activity concentrations with proteins levels, respectively. SA: specific activity; AC: activity concentration.

supported that HIU levels do not achieve steady-state within $100 \mathrm{~s}$ since reaction initiation. The interference from HIU with uric acid absorbance in Tris- $\mathrm{HCl}$ buffers was alleviated by the increase in reaction $\mathrm{pH}$ or the use of magnesium ion (Additional file 2: Figure S1, S2). After reaction initiation at $103 \mathrm{U} / \mathrm{L}$ uricase, there were no detectable increases in absorbance at $293 \mathrm{~nm}$ but tiny increases in absorbance at $308 \mathrm{~nm}$ with $0.12 \mathrm{mM}$ uric acid in Tris- $\mathrm{HCl}$ buffer at $\mathrm{pH} 7.4$ (Additional file 2: Figure S3), and there were also no increases in absorbance at $308 \mathrm{~nm}$ with $0.30 \mathrm{mM}$ uric acid in $0.20 \mathrm{M}$ sodium borate buffers at $\mathrm{pH} 7.4$ (Figure 1); those results supported the interference from HIU with uric acid absorbance is weaker at $293 \mathrm{~nm}$ than at $308 \mathrm{~nm}$ and is even negligible in borate buffers at both wavelengths. At $400 \mathrm{U} / \mathrm{L}$ uricase, uric acid concentration after the lag time of $20 \mathrm{~s}$ in the borate buffer at $\mathrm{pH} 7.4$ was smaller than $0.27 \mathrm{mM}$, but there were still no increases in absorbance at $308 \mathrm{~nm}$. No increases in absorbance at $308 \mathrm{~nm}$ during uricase reaction were detected in borate buffers at higher $\mathrm{pH}$. On the other hand, when activities of the recombinant uricase were decreasing, $K_{\mathrm{m}}$ by

Table 2 ROC analysis of activities in lysates $(n=30)$

\begin{tabular}{cccccc}
\hline $\begin{array}{c}\text { Candidate } \\
\text { pairs }\end{array}$ & $\begin{array}{c}\text { Ratios after } \\
\text { purification }\end{array}$ & $\begin{array}{c}\text { Ratio } \\
\text { by AC }\end{array}$ & $\begin{array}{c}\text { AUC } \\
\text { by AC }\end{array}$ & $\begin{array}{c}\text { Ratio } \\
\text { by SA }\end{array}$ & $\begin{array}{c}\text { AUC } \\
\text { by SA }\end{array}$ \\
\hline A-B & 1.37 & 1.07 & 0.559 & 1.19 & 0.799 \\
B-C & 1.58 & 1.54 & 0.895 & 1.56 & 0.985 \\
A-C & 2.15 & 1.67 & 0.921 & 1.82 & 1.000 \\
C-D & 1.90 & 5.45 & 1.000 & 5.60 & 1.000 \\
B-D & 3.00 & 8.48 & 1.000 & 8.60 & 1.000 \\
A-D & 4.10 & 9.09 & 1.000 & 9.92 & 1.000 \\
\hline
\end{tabular}

Based on error propagation, the experimental ratios in pairs had CVs about $20 \%$. SA: specific activity; AC: activity concentration. measuring absorbance at $308 \mathrm{~nm}$ in borate buffers decreased to stable values (Figure 2). The use of a higher reaction $\mathrm{pH}$ enabled the estimation of such a stable $K_{\mathrm{m}}$ at higher uricase activities. For Asahi-Kasei uricase with $K_{\mathrm{m}}$ of about $30 \mu \mathrm{M}$, the highest uric acid levels were below $0.10 \mathrm{mM}$ so that uric acid absorbance can be measured at $293 \mathrm{~nm}$ in borate buffers to determine initial rates for estimating its $K_{\mathrm{m}}$; there were no changes of $K_{\mathrm{m}}$ over a wide range of uricase activities. Moreover, by measuring absorbance at $293 \mathrm{~nm}$ with $0.075 \mathrm{mM}$ uric acid in borate buffer at $\mathrm{pH}$ 9.2, initial rates linearly responded to uricase quantities ranging from 0.6 to about $12 \mathrm{U} / \mathrm{L}$ when absorbance was monitored at 5 -s intervals. Repetitive assays for uricase activities from 3.0 $\mathrm{U} / \mathrm{L}$ to $12 \mathrm{U} / \mathrm{L}$ with a pooled lysate showed CVs below $5.5 \%(n=11)$.

Taken together, the assay of absorbance of uric acid at $293 \mathrm{~nm}$ in alkaline borate buffers is reliable for measuring uricase initial rates within a reasonable range.

\section{Recognition of a mutant of higher activity}

To recognize positive candidates in a library, a decision method should have sensitivity and specificity as high as possible. One-fold or $100 \%$ improvement of catalytic capacity of a mutant already makes sense and the recognition of such a mutant is a challenge. Hence, four uricases with ratios of catalytic capacities from about 1.3 -fold to about 4-fold were employed as models to test the potential solutions to the challenge.

The abundance of uricase proteins in lysates was examined at first. With the same quantities of total proteins for analyses, band density of four uricases was different after SDS-PAGE followed by staining of protein bands, or after PAGE followed by staining of uricase activity (Figure 3 ). The averages of total proteins in

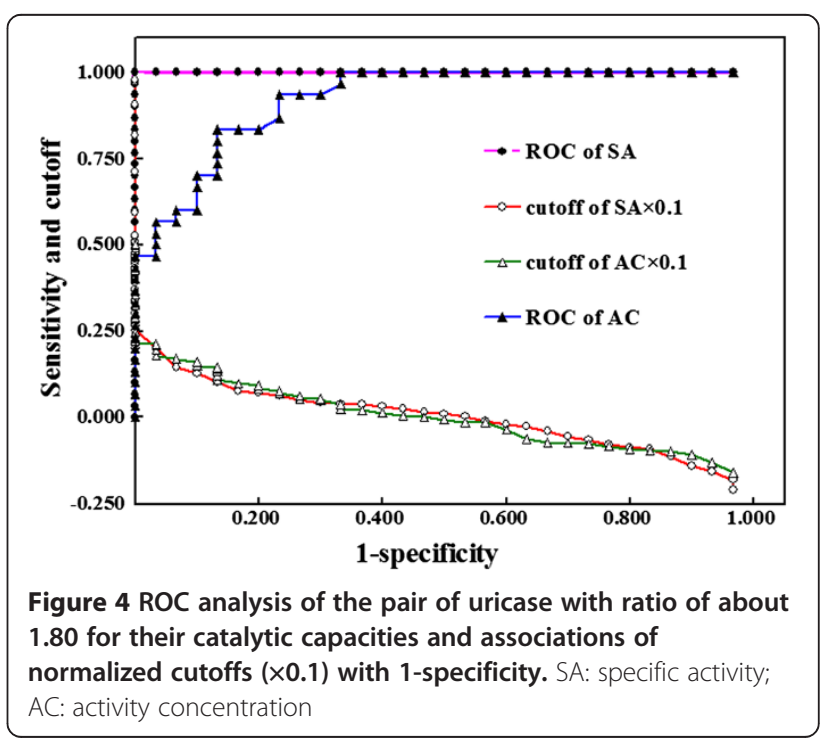


lysates of four uricase mutants had no statistical differences, but their activity concentrations displayed positive associations with the levels of total proteins in lysates; those results supported the concern on negative impacts of variations of the levels of mutant proteins in lysates on the recognition of positive candidates. Activity concentration of uricases in lysates basically followed normal distribution, but specific activities of most candidates displayed non-normal distribution (Table 1). Candidate D had the lowest abundance and the lowest specific activity. The ratios of specific activities among candidate $\mathrm{A}, \mathrm{B}$ and $\mathrm{C}$ showed no significant deviations from those of catalytic capacities, but the ratios of specific activities of candidate $\mathrm{A}, \mathrm{B}$ and $\mathrm{C}$ to that of candidate $\mathrm{D}$ were more than twice of those of catalytic capacities, respectively (Table 2). Such large deviations in ratios of specific activities of the three uricase mutants to candidate $\mathrm{D}$ from those of their catalytic capacities should be associated with the lower abundance of candidate D in its lysate. Hence, the variations in the abundance of mutant proteins in lysates should be considered carefully.

To reveal the differences between the comparison of activity concentration and specific activity for recognizing positive candidates, ROC analyses were performed [40,41]. Clearly, the smaller differences in catalytic capacities of paired uricases led to smaller AUCs by comparing either activity concentration or specific activity (Table 2). When ratios of catalytic capacities of a pair of uricases were over 3.0, the comparison of either activity index consistently yielded AUCs of 1.000. For uricase pairs with ratios of their catalytic capacities below 2.0, the comparison of specific activity gave higher AUCs than the comparison of activity concentration (Table 2). Hence, for screening uricase mutants, the comparison of specific activity calculated from the levels of total proteins in lysates is favorable for recognizing positive candidates of small improvement of activity.

By the comparison of specific activity to recognize a positive candidate, a practical threshold is required. The ratio of specific activity between candidate $\mathrm{A}$ and $\mathrm{C}$ was over 1.7 with either crude enzymes or purified enzymes (Additional file 1: sheet 1: mutant sequences and summary), and their activity concentrations and specific activities exhibited consistent CVs (Table 1). The practical threshold for the recognition of a positive candidate was investigated with this pair of uricases. For ROC analysis of the recognition of candidate $\mathrm{A}$ as a positive candidate against candidate $\mathrm{C}$, the deviations of the preset cutoffs from the mean of the starting material were divided by the SD of the starting material for plotting against 1-specificty to observe the association of decision sensitivity with the preset cutoffs (Figure 4). For no less than $90 \%$ specificity to recognize the positive candidate in this uricase pair, the mean of specific activity plus 1.4-fold of the SD of the starting material can be a practical threshold (Additional file 1: sheet 2: ROC analysis of activity). By using such a threshold to other uricase pairs, the comparison of specific activity always gave higher sensitivity to identify positive candidates than the comparison of activity concentration. Hence, the comparison of specific activity calculated from the levels of total proteins against a suitable threshold is more favorable for recognizing positive candidates with small improvement of catalytic capacity.

\section{Conclusion}

For screening uricase mutants, the assay of UV absorbance of uric acid at $293 \mathrm{~nm}$ is effective to measure uricase activities within a reasonable range in alkaline borate buffers. To recognize a mutant of small improvement of catalytic capacity, specific activity calculated from total proteins in a lysate is an index better than activity concentration; a practical threshold for comparison to recognize a positive candidate can be the mean plus 1.4-fold SD of specific activities of the starting material.

\section{Additional files}

Additional file 1: The following additional data are available with the online version of this paper. Sheet 1: mutant sequences and summary; Sheet 2: ROC analysis of activity.

Additional file 2: The following additional data are available with the online version of this paper. Figure S1. Effects of magnesium ion on the interference from $\mathrm{HIU}$ in Tris- $\mathrm{HCl}$ buffer at pH 7.4; Figure S2. Effects of magnesium ion on the interference from $\mathrm{HIU}$ in Tris- $\mathrm{HCl}$ buffer at pH 8.9; Figure S3. Interference from HIU with $A_{293}$ and $A_{308}$ for uric acid in Tris- $\mathrm{HCl}$ buffer at $\mathrm{pH} 7.4$.

\section{Abbreviations}

AUC: Area-under-the-curve; BSA: Beef serum albumin; HIU: 5-hydroisourate; IPTG: Isopropyl- $\beta-D$-thiogalactoside; $K_{m}$ : Michaelis-Menten constant; OVA: Ovalumin; ROC: Receiver-operation-curve; Tris: Tri-(hydroxylmethyl)aminomethane.

\section{Competing interest}

The authors declared no competing interest.

\section{Authors' contributions}

$J F, H L, A G, L F, G L, J P$ and $Y L$ performed the experiments; $F L, J F, H L$ and $X Y$ analyzed experimental data; $X Y$ and $F L$ wrote the manuscript; $F L$ and $X Y$ conceived the idea. All authors read and approved the final manuscript.

\section{Acknowledgment}

Supported by Natural Science Foundation Project of CQ (CSTC2011BA5039), National Natural Science Foundation of China (nos. 30672009, 81071427), High-Technology Program of China (No. 2011AA02A108), and program for New Century Excellent Talent in University (NCET-09-0928). Miss Liping Feng is currently an undergraduate student at College of Laboratory Medicine in Chongqing Medical University.

Received: 18 December 2012 Accepted: 6 March 2013 Published: 17 April 2013 


\section{References}

1. Bershtein S, Tawfik DS: Advances in laboratory evolution of enzymes. Curr Opin Chem Biol 2008, 12:151-158.

2. Turner NJ: Directed evolution drives the next generation of biocatalysts. Nat Chem Biol 2009, 5:567-573

3. Jackel C, Hilvert D: Biocatalysts evolution Curr Opin Biotechnol 2010, 21:753-759.

4. Zhang C, Yang XL, Yuan YH, Pu J, Liao F: Site-specific PEGylation of therapeutic proteins via optimization of both accessible reactive amino acid residues and PEG derivatives. BioDrugs 2012, 26:209-215.

5. Jochens $H$, Bornscheuer UT: Natural diversity to guide focused directed evolution. Chembiochem 2010, 11:1861-1866.

6. Hulley ME, Toogood HS, Fryszkowska A, Mansell D, Stephens GM, Gardiner JM, Scrutton NS: Focused directed evolution of pentaerythritol tetranitrate reductase by using automated anaerobic kinetic screening of site-saturated libraries. Chembiochem 2010, 11:2433-2447.

7. Mazura P, Filipi T, Soucek P, Brzobohaty B: Focused directed evolution of $\beta$-glucosidases: theoretical versus real effectiveness of a minimal working setup and simple robust screening. Carbohydr Res 2011, 346:238-242.

8. Feng X, Sanchis J, Reetz MT, Rabitz H: Enhancing the efficiency of directed evolution in focused enzyme libraries by the adaptive substituent reordering algorithm. Chemistry 2012, 18:5646-5654

9. Brideau C, Gunter B, Pikounis B, Liaw A: Improved statistical methods for hit selection in high-throughput screening. J Biomo/ Screen 2003, 8:634-647.

10. Malo N, Hanley JA, Cerquozzi S, Pelletier J, Nadon R: Statistical practice in high-throughput screening data analysis. Nat Biotechnol 2006, 24:167-175.

11. Zhang XD: A new method with flexible and balanced control of false negatives and false positives for hit selection in RNA interference highthroughput screening assays. J Biomo/ Screen 2007, 12:645-55.

12. Zhang XD, Ferrer M, Espeseth AS, Marine SD, Stec EM, Crackower MA, Holderm DJ, Heyse JF, Strulovici B: The use of strictly standardized mean difference for hit selection in primary RNA interference high-throughput screening experiments. J Biomol Screen 2007, 12:497-509.

13. Zhang XD: Illustration of SSMD, z score, SSMD*, $\mathrm{z}^{*}$ score, and t statistic for hit selection in RNAi high-throughput screens. J Biomol Screen 2011, 16:775-85.

14. Richette P, Bardin T: Gout. Lancet 2010, 375:318-328.

15. Schlesinger N, Yasothan U, Kirkpatrick P: Pegloticase. Nat Rev Drug Discov 2011, 10:17-18.

16. Sherman MR, Saifer MG, Perez-Ruiz F: PEG-uricase in the management of treatment-resistant gout and hyperuricemia. Adv Drug Deliv Rev 2008, 60:59-68.

17. Terkeltaub R: Update on gout: new therapeutic strategies and options. Nat Rev Rheumatol 2010, 6(1):30-38.

18. Zhao YS, Yang XY, Lu W, Liao H, Liao F: Uricase based methods for determination of uric acid in serum. Microchim Acta 2009, 164:1-6.

19. Ganson NJ, Jelly KS, Scarlett E: Control of hyperuricemia in subjects with refractory gout, and induction of antibody against poly(ethylene glycol) (PEG), in a phase I trial of subcutaneous PEGylated urate oxidase. Arthritis Res Ther 2005, 8:R12-18.

20. Sundy JS, Ganson NJ, Kelly SJ, Scarlett EL, Rehrig CD, Huang W, Hershfield MS: Pharmacokinetics and pharmacodynamics of intravenous PEGylated recombinant mammalian urate oxidase in patients with refractory gout. Arthritis Rheum 2007, 56:1021-1028.

21. Yang XL, Yuan YH, Zhan CG, Liao F: Uricases as therapeutic agents to treat refractory gout: current states and future directions. Drug Dev Res 2012, 73:66-72.

22. Sarma AD, Tipton PA: Evidence for urate hydroperoxide as an intermediate in the urate oxidase reaction. J Am Chem Soc 2000, 122:11252-11253.

23. Larsen T, Moyes KM: Fluorometric determination of uric acid in bovine milk. J Dairy Res 2010, 77:438-444.

24. Luo W, Li YS, Yuan J, Zhu L, Liu Z, Tang H, Liu S: Ultrasensitive fluorometric determination of hydrogen peroxide and glucose by using multiferroic BiFeO(3)nanoparticles as a catalyst. Talanta 2010, 81:901-907.

25. Huang SH, Wu TK: Modified colorimetric assay for uricase activity and a screen for mutant Bacillus subtilis uricase genes following StEP mutagenesis. Eur J Biochem 2004, 271:517-523.

26. Imhoff RD, Power NP, Borrok MJ, Tipton PA: General base catalysis in the urate oxidase reaction: evidence for a novel thr-lys catalytic diad. Biochem 2003, 42:4094-4100.

27. Wolf SP: Ferrous ion oxidation in presence of ferric ion indicator xylenol orange for measurement of hydroperoxides. Methods Enzymol 1994 233:182-189.
28. Chinh NH: Mechanism of interference by uric acid in the glucose oxidase/peroxidase method for serum glucose. Clin Chem 1974, 20:499-501.

29. Fleming JK, Gadsden RH, Kabbani I: Definitive characterization of uric acid as an interferent in peroxidase indicator reactions and a proposed mechanism of action. Clin Biochem 1988, 21:27-32.

30. Priest $D G$, Pitts OM: Reaction intermediate effects on the spectrophotometric uricase assay. Anal Biochem 1972, 50:195-205.

31. Kahn K, Tipton PA: Spectroscopic characterization of intermediates in the urate oxidase reaction. Biochemistry 1998, 37:11651-11659.

32. Zhao YS, Zhao LN, Yang GQ, Tao J, Liao F: Characterization of a uricase from Bacillus fastidious A.T.C.C. 26904 and its application to serum uric acid assay by a patented kinetic uricase method. Biotechnol Appl Biochem 2006, 45:75-80.

33. Zhang C, Yang XL, Feng J, Yuan YH, Li X, Bu YQ, Xie YL, Yuan HD, Liao F: Effects of modification of amino groups with poly(ethylene glycol) on a recombinant uricase from Bacillus fastidiosus. Biosci Biotechnol Biochem 2010, 74:1298-1301.

34. Feng J, Li X, Yang XL, Zhang C, Yuan YH, Pu J, Zhao YS, Xie YL, Yuan HD, $\mathrm{Bu} Y \mathrm{Q}$, Liao F: A new practical system for evaluating pharmacological properties of uricase as potential drug to handle hyperuricemia. Arch Pharm Res 2010, 33:1761-1769.

35. Liao F, Zhu XY, Wang YM, Zuo YP: The comparison of the estimation of enzyme kinetic parameters by fitting reaction curve to the integrated Michaelis-Menten rate equations of different predictor variables. J Biochem Biophys Methods 2005, 62:13-24.

36. Liu BZ, Zhao YS, Zhao LN, Xie YL, Zhu S, Li ZR, Liu Y, Lu W, Yang XL, Xie GQ, Zhong HS, Yu MA, Liao H, Liao F: An integration strategy to estimate the initial rates of enzyme reactions with much expanded linear ranges using uricases as models. Anal Chim Acta 2009, 631:22-28.

37. Liao F, Yang DY, Tang JQ, Yang XL, Liu BZ, Zhao YS, Zhao LN, Liao H, Yu MA: The measurement of serum cholinesterase activities by an integration strategy with expanded linear ranges and negligible substrate-activation. Clin Biochem 2009, 42:926-928.

38. Bradford MM: A rapid and sensitive method for the quantitation of microgram quantities of protein utilizing the principle of protein-dye binding. Anal Biochem 1976, 72:248-254.

39. Zhao YS, Yang XL, Li XY, Bu YQ, Deng P, Zhang C, Feng J, Xie YL, Zhu S, Yu MA, Liao F: Reversible inactivation of an intracellular uricase from Bacillus fastidiosus via dissociation of homotetramer into homodimers in solutions of low ionic strength. Biosci Biotechnol Biochem 2009, 73:2141-2144.

40. Langlotz CP: Fundamental measures of diagnostic examination performance: usefulness for clinical decision making and research. Radiology 2003, 228(1):3-9.

41. Alonzo TA, Pepe MS: Development and evaluation of classifiers. Methods Mol Biol 2007, 404:89-116.

doi:10.1186/1752-153X-7-69

Cite this article as: Feng et al:: Comparison of activity indexes for recognizing enzyme mutants of higher activity with uricase as model. Chemistry Central Journal 2013 7:69.

\section{Publish with ChemistryCentral and every scientist can read your work free of charge \\ "Open access provides opportunities to our colleagues in other parts of the globe, by allowing anyone to view the content free of charge." W. Jeffery Hurst, The Hershey Company. \\ - available free of charge to the entire scientific community \\ - peer reviewed and published immediately upon acceptance \\ - cited in PubMed and archived on PubMed Central \\ - yours - you keep the copyright \\ Submit your manuscript here: \\ http://www.chemistrycentral.com/manuscript/<smiles>c1ccccc1</smiles> \\ Chemistry Central}

